\title{
Feed Signal Influence and Potential Performances of a Compact Radiation Source Based on a Helical Antenna
}

\author{
Laurent Pecastaing1, Marc Rivaletto' ${ }^{1}$ Antoine de Ferron' ${ }^{1}$, Romain Pecquois ${ }^{2}$, \\ René Vézinet $^{3}$, Jean-Christophe Diot ${ }^{3}$, Stéphane Tortel ${ }^{3}$ \\ ${ }^{1}$ Laboratoire SIAME, Equipe Génie Electrique, Université de Pau et des Pays de I'Adour, Hélioparc Pau-Pyrénées, \\ Pau, France \\ ${ }^{2} \mathrm{Hi}$-Pulse, 300 rue des arts et métiers, Pont-de-Pany, France \\ ${ }^{3}$ French Alternative Energies and Atomic Energy Commission (CEA), Gramat, France \\ Email: laurent.pecastaing@univ-pau.fr
}

Received 8 April 2015; accepted 14 July 2015; published 17 July 2015

Copyright $@ 2015$ by authors and Scientific Research Publishing Inc.

This work is licensed under the Creative Commons Attribution International License (CC BY).

http://creativecommons.org/licenses/by/4.0/

c) (i) Open Access

\begin{abstract}
In the class of emerging high power electromagnetic sources, a complete pulsed power source, named MOUNA (French acronym of "Module Oscillant Utilisant une Nouvelle Architecture") has been developed. This device must transmit waveforms with a wide frequency band and a high figure-of-merit. To improve the overall performance of the MOUNA system while maintaining its compact size, two approaches are being explored in the paper: the replacement of the dipole antenna by a helical antenna and its feeding signal influence. Helical antenna is cylindrical shape and relatively compact. It offers relatively good gain factor and directivity. The waveform delivered to the antenna is directly related to the amplitude of the radiated electric field. Therefore, different waveforms (step pulse, Gaussian pulse, bipolar pulse and damped sinusoid) are compared to point out the feed signal influence on the radiated electric field. Switch oscillators appear to be considered as interesting resonant sources for driving an antenna. The novel radiating source consists of a primary power source, a resonant transformer, a coaxial transmission line damped oscillator (also termed as coaxial resonator), and a helical antenna. This high voltage pulsed source is very compact (volume of only $2500 \mathrm{cc}$ without the antenna). Our study aims at designing the antenna (number of turns, size...) and a coaxial damped oscillator directly implemented at the output of the transformer. A CST-based simulation is proposed to predict the performances of this wideband source.
\end{abstract}

\section{Keywords}

Pulse Transformer, Helical Antenna, Coaxial Damped Oscillator, High Voltage, Peaking Switch, Electric Field 


\section{Introduction}

High power microwave (HPM) sources have been developed over the past few decades for many applications, including defense for disrupting electronically-controlled system, medical for cell manipulation, and biological for food and water purification [1]. Defense requirements are the most prevalent. Ultra-Wide Band (UWB) source, one of the HPM sources, can be used for target identification, buried objects detection, radar system and directed energy weapon system [2]-[7]. Intentional electromagnetic interference describes a wide range of technologies that are designed to disrupt the performance of electronic systems by introduction of spurious signals in different frequency ranges. These systems must also typically be high power, since it is generally desired to have as large an effect possible at as long distances possible [8]. Even if the radiated energy of a single pulse UWB system is low, these systems can disrupt, disable, or destroy electronic target because of the repetitive operation. In principle, for affecting any target with such radiation, the frequency has to be matched to the frequency absorption spectrum of the target [7]. However, the main advantage of pulsed wideband radiation, with pulse durations in the nanosecond range, is that it is very aggressive for systems with wiring [9] [10].

There are a number of high power microwave efforts, and an equal number of antenna geometries being pursued. Example geometries generally include traditional TEM horns, traveling wave antenna [3] [4], the Impulse Radiating Antenna (IRA) [2], dipoles and bicones [9]-[11], among others [12]. However, most of these geometries are either large and not deployable, or radiate with undesirable radiation pattern. Furthermore, generating very large electric field strengths is difficult, since extremely high voltages are required. Insulating the high voltage increases the complexity of the overall source design, and typically results in larger volumes.

A previous work supported by the DGA aimed at developing an extremely compact source [11]. The electromagnetic pulse source named MOUNA is composed of a set of batteries, a DC/DC converter to charge four capacitors, four synchronized spark gap switches, a resonant transformer generating $600 \mathrm{kV} / 265 \mathrm{~ns}$ pulses, an oil peaking switch and a dipole antenna. This source is compact, with dimensions less than $1 \mathrm{~m}$ in length and $22 \mathrm{~cm}$ in diameter. These dimensions correspond to a 3800 cc volume. However, to radiate very high electric fields, the low gain of the dipole antenna is detrimental. The use of a directional antenna may improve performance really significantly focusing the radiation in a preferred direction. The main characteristics of the axial helical antenna (compactness, high gain on the axis, wideband spectrum and high impedance) make it an excellent candidate. Helical antennas are being studied for high power radiofrequency applications [12]. The helical geometry is very appealing for it is relatively small and conformal geometry, which makes it suitable for air platforms. Furthermore, the antenna is very directive, with typical beam widths of 20 - 40 degrees, and offering relatively good values of gain, from 8 - $15 \mathrm{~dB}$.

Some sources already exist and use a Marx generator associated to a helical antenna [13]-[15]. Only one associates a $30 \mathrm{kV}$ pulse transformer, a damped oscillator and a helical antenna [13]. Our structure uses also an ultra-compact high voltage resonant transformer which will allow an important volume gain versus other topologies (particularly Marx generators used more conventionally).

The waveform of the antenna pulse feeding is of importance for the overall source performance. Step pulse, bipolar pulse, Gaussian pulse and damped sinusoid are studied in the paper. One of the typical moderate band source is the damped sinusoidal system which employs a self breaking switch and antenna at each end of high voltage transmission line. The waveform delivered to the antenna will be characterized by one or more damped sinusoids. The pulse width (or number of cycles to some fraction of the peak) depends on the antenna impedance and other parasitic effects (non ideal switch, etc.). When the switch operates, the system generates a damped sine signal into an antenna. The system radiates $\mathrm{N}$ cycles of the same oscillation frequency wave with amplitude damp down. Radiated energy of damped sinusoid system might be more than UWB source, because duration of the former is longer than the one of the latter.

Including the transformer, the first stages principle is conserved to build the pulsed power generator of the new helical radiating system (Figure 1). The oil switch and the radial line of the previous version of the source are replaced by a multichannel annular gas switch [16] [17] associated to a coaxial quarter wave oscillator [18]-[20]. A resonant transformer is used to feed $500 \mathrm{kV}$ high voltage pulse to the coaxial damped oscillator. This oscillator feeds a $140 \Omega$ helical antenna.

Principle and/or realization of switched oscillators are described in many papers [18]-[20] where the line has a radial section and a coaxial section due to the use of only one switch. Our oscillator is only composed of a coaxial section switched by the multichannel annular gas switch. The design of the novel radiating source com- 


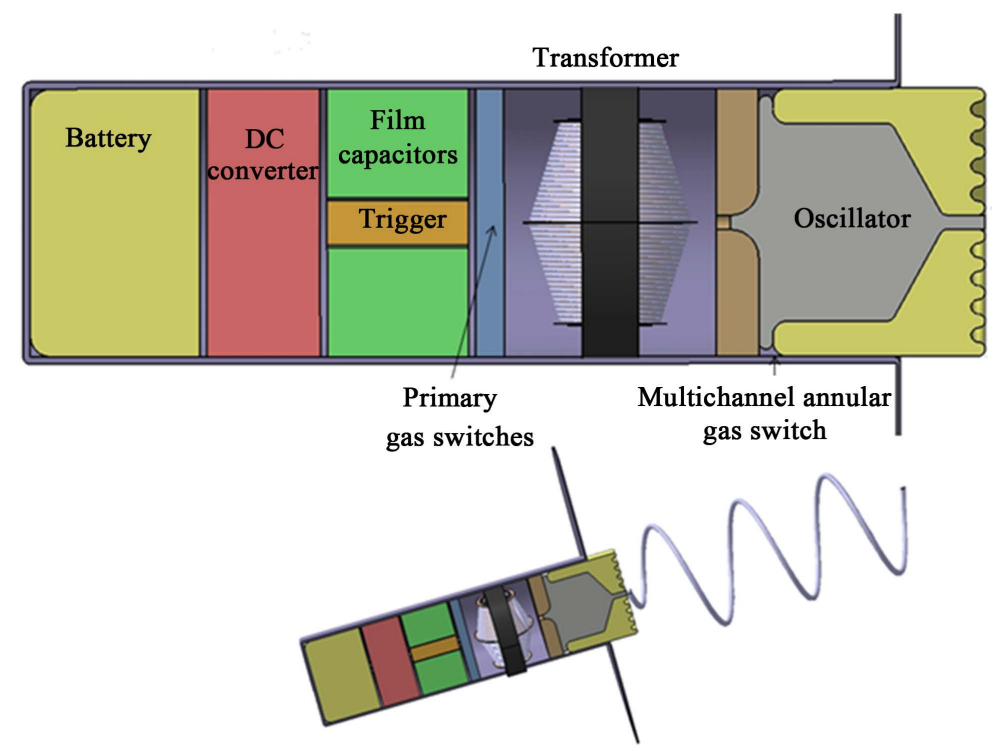

Figure 1. Arrangement of the new helical antenna radiating system.

posed of the MOUNA pulsed power source, the switch oscillator and the helical antenna is also described and the predicted overall performances of the electrical characteristics are detailed.

\section{Helical Antenna Design}

\subsection{Antenna Design}

The principle of a helical antenna was described for the first time by J.D. Kraus [12] (Figure 2). In these works, Kraus establishes relations between the dimensions of the antenna for which the field is a maximum in the direction of helix axis and is circularly polarized: the axial mode of radiation. If the helix circumference is comparable to the wavelength, the antenna operates as a directional antenna radiating a beam off the ends of the helix, along the antenna's axis. It radiates circularly polarized waves.

If the wavelength $(\lambda)$ is the free-space wavelength of the radiating field, these relations follow:

$$
\begin{gathered}
C=\pi D \approx \lambda \\
\alpha=\tan ^{-1}=\tan ^{-1} \frac{S}{\pi D} \approx 12.5^{\circ} \\
D_{\text {ground }} \approx \frac{3 \lambda}{4}
\end{gathered}
$$

and the antenna input impedance $R$ is given by:

$$
R \approx 140 \times\left(\frac{C}{\lambda}\right) \approx 140 \Omega
$$

The design of a simple helical antenna follows from these equations, in which the circumference of the helix is one wavelength so that diameter $D$ equals $\lambda / \pi$; the length of the helix is given by $N \cdot \lambda / 4$, where $N$ is the number of helix turns and $\lambda / 4$ is the turn-to-turn spacing. The ground plane diameter $(D)$ is approximately $3 / 4 \lambda$. The pitch angle which controls how far the helix antenna grows in the z-direction per turn is given by $\alpha$ where $S$ is the vertical spacing between turns for the antenna.

The MOUNA source radiates around $200 \mathrm{MHz}$, if we do not change this frequency, the helical antenna diameter will be around $48 \mathrm{~cm}$. So, the MOUNA diameter $(22 \mathrm{~cm})$ will be increased drastically and its compactness will be altered. To keep MOUNA dimensions, the helical antenna diameter must be around $22 \mathrm{~cm}$, in free-space the wavelength of the radiating field must be around $69 \mathrm{~cm}$ and its frequency around $434 \mathrm{MHz}$. That is why the axial mode helical antenna is designed to operate at a frequency around $434 \mathrm{MHz}$. So, the antenna mechanical characteristics are summarized in the Table 1. 


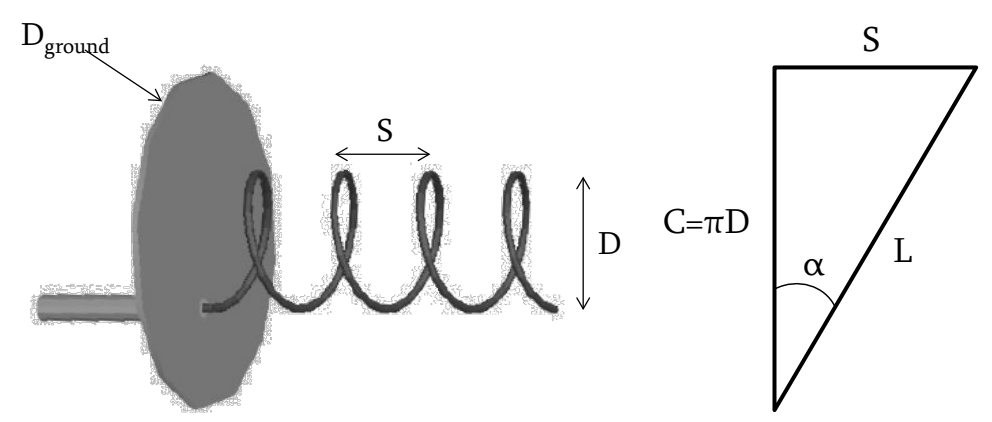

Figure 2. Geometric features of an axial helical antenna. $D$ : Diameter of helix (m); C: Circumference of helix (m); $S$ : Spacing between turns (m); $D_{\text {ground: }}$ Diameter of the reflector (m); $\alpha$ : pitch angle (degrees); $L$ : length of turn (m); $N$ : number of turns.

Table 1. Antenna mechanichal design.

\begin{tabular}{cc}
\hline Diameter $D$ & $22.0 \mathrm{~cm}$ \\
Spacing $S$ & $15.3 \mathrm{~cm}$ \\
Reflector diameter $D_{\text {ground }}$ & $51.8 \mathrm{~cm}$ \\
Number of turns & 3 \\
\hline
\end{tabular}

So, a new damped oscillator must be designed. The MOUNA radial output line will be replaced by a coaxial quarter wave oscillator sized to oscillate at $434 \mathrm{MHz}$.

\subsection{Feed Signal Influence}

In [18] Giri et al. describe the problem of designing switched oscillators and their integrations into a helical antenna. This paper is following this track. But a few realizations, described in [13] [14], do not use a damped sinusoidal signal to feed the helical antenna. So, it was interesting to study the influence of the feed signal on the field intensity produced by the antenna. A CST Microwave Studio simulation of a 3 turn helical antenna model is computed.

For simulation, four different normalized voltages are applied on a $140 \Omega$ adapted discrete port localized on the antenna input. The four signals are a step pulse (pink), a Gaussian pulse (green), a bipolar pulse (blue) and a $434 \mathrm{MHz}$ damped sinusoid (red) (Figure 3).

The simulation provides the following results (Figure 4). These results clearly show a strong dependence of the shape of the radiated field with the shape of the supply signal. The antenna factor (i.e. $\mathrm{rE} / \mathrm{V}$ ) also varies greatly (Table 2). The highest antenna factor (0.82) is obtained when the antenna is fed by a $434 \mathrm{MHz}$ damped sinusoid. Interesting results (0.6) are also noted in the case of a bipolar pulse.

\section{Coaxial Damped Oscillator Design}

Many papers [16]-[18] describe the principle and realization of a low impedance line damped oscillator to feed a high impedance antenna. In most cases, the quarter wave line is composed of a radial section followed by a coaxial section. Unlike other works where the transmission line oscillator consists of radial transmission line section and coaxial transmission line section, our oscillator design is simplified. The design of the coaxial section follows from simple coaxial transmission line theory.

According to a low impedance quarter wave transmission line oscillator, the transmission line is charged to a high voltage. A switch is located at one end of the transmission line and antenna is placed at the other end of the transmission line. By charging a transmission line to some high voltage and then shorting it by some fast-closing switch, a resonant waveform is applied to an antenna at the other end: a damped sinusoid oscillation is generated to the antenna. Indeed, when the oscillator is switched, the circuit is equivalent to a damped oscillator (high impedance of the transformer at its input and low impedance of the switch at its output) from which its energy is radiated by the helical antenna. 


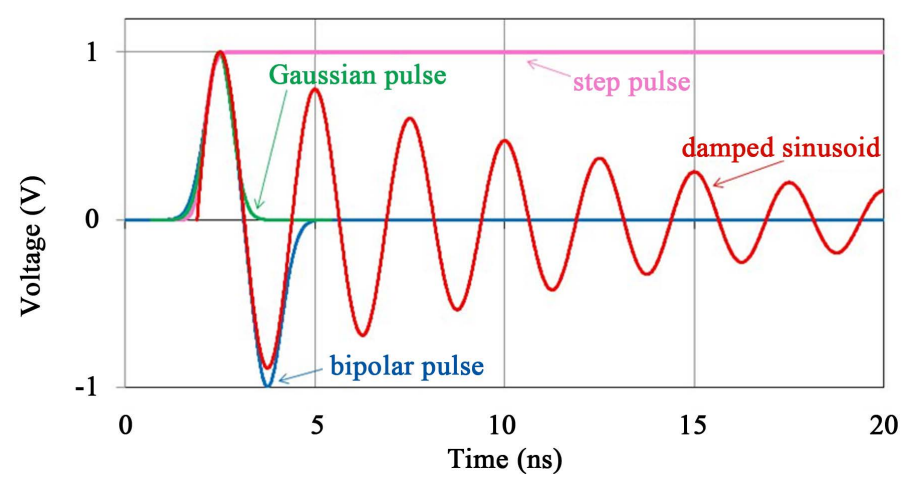

Figure 3. Normalized signals applied to the antenna port.

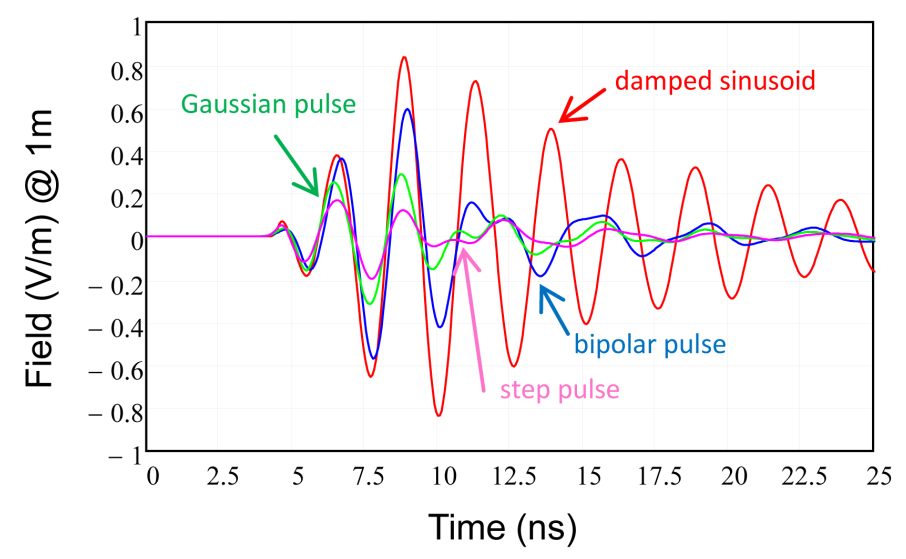

Figure 4. Radiated fields for the step pulse (pink), the Gaussian pulse (green), the bipolar pulse (blue) and the $434 \mathrm{MHz}$ damped sinusoid (red).

Table 2. Variations of the antenna factor.

\begin{tabular}{cc}
\hline Supply signal & Antenna factor \\
\hline Step pulse & 0.2 \\
Gaussian pulse & 0.32 \\
Bipolar pulse & 0.6 \\
Damped sinusoid & 0.8 \\
\hline
\end{tabular}

Due to the presence of two quite different sections, the design and the realization of this line oscillator is very accurate [15]. Design must be simplified if we can propose only a coaxial line oscillator with polyamide 6 dielectric (PA6). The volume of the oscillator is filled with this polymer. This choice allows the feeding of the line through the switch side. The need for a fast switch is clear. A real switch will have some inductance and resistance which will modify the resonance somewhat. The switch will need to be physically small compared to the transmission line length. To keep only one switch, this solution requires using a multichannel annular gas switch which will reduce the switch inductance. Its time of breakdown will be drastically reduced in high pressurized nitrogen atmosphere [21].

To keep the transformer performances (maximum output voltage: $600 \mathrm{kV}$, rise time: $265 \mathrm{~ns}$, Figure 5), the capacitance of the coaxial line oscillator must remain close to $80 \mathrm{pF}$ due to the resonance conditions of the transformer. This measurement (Figure 8) was provided by a Half-Megavolt capacitive probe developed in common by Université de Pau, Loughborough University (UK) and the North star company [22]. Its features are: attenuation, 1/10.000; input capacitance, 12.4 pF; maximum voltage, $620 \mathrm{kV}$; bandwidth, $8 \mathrm{~Hz}$ - $55 \mathrm{MHz}$; accuracy, $<4 \%$ in a volume of only 1500 cc. 


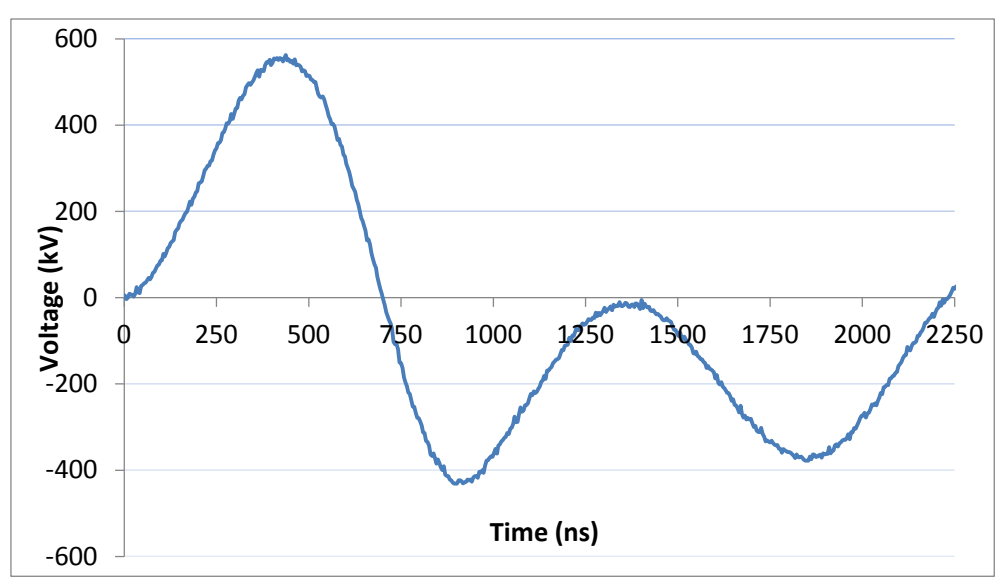

Figure 5. Typical output transformer voltage (input $9 \mathrm{kV}$, output $560 \mathrm{kV}$ ).

Insulation rules impose $18 \mathrm{~cm}$ for the outer diameter $\left(D_{\text {out }}\right)$ maximum value. Indeed the geometry of the switch oscillator system must satisfy the static requirements for the self-breakdown switch operation in addition to the geometric constraint that define the resonance parameters of the system. Therefore, to oscillate at 434 $\mathrm{MHz}$, the main characteristics of the line oscillator are summarized in the Table 3.

The length of the transmission line should be $9.11 \mathrm{~cm}$ because of the center frequency. The designed characteristic impedance and average switching voltage of the coaxial damped oscillator is $7.3 \Omega$ and $500 \mathrm{kV}$ respectively. The output of the high voltage transformer is fed to the transmission line coaxial damped oscillator which is designed to oscillate at center frequency of $434 \mathrm{MHz}$.

\section{Coaxial Oscillator LTspice and CST Simulations}

Prior to detailed design and manufacturing of the coaxial damped oscillator, the electrical parameters and performances of transmission line oscillator were simulated using electronic and electromagnetic simulation tools. The programs for the simulation activity are LTspice, and CST Microwave studio. The LTspice tool is used to simulate the oscillation frequency, and we assumed the uniform $7.3 \Omega$ coaxial transmission line section is connected with the $140 \Omega$ antenna.

Using predicted values above, an LTspice-based study of the line damped oscillator is realized (Figure 6).

(Rantenna is the impedance of the helical antenna considered as a constant for the electric simulation)

On Figure 7, the simulation results of the damped oscillator voltage are presented assuming that the breakdown time of the annular switch is about 500 ps. The simulation confirms calculations.

For the same conditions and dimensions, a CST Microwave Studio simulation of the damped oscillator is computed. For simulation, a $1 \mathrm{~V}$ normalized voltage is applied on an adapted discrete port localized on the multichannel gas annular gas switch (Figure 8).

The simulation provides the following results (Figure 9). The results are similar in the LTspice and CST simulations. In the results of the two types of simulation (Figure 7 and Figure 9), the frequency is close to the expected $434 \mathrm{MHz}$.

\section{Novel Multi Primary Winding Resonant High-Voltage Pulsed Transformer}

As explained in Section 2, to keep the dimensions of the MOUNA source, the number of turns of the helical antenna is 3 . The voltage signal present on Figure 9 is applied to a 3 turn helical antenna simulated on CST. For simulation, this $1 \mathrm{~V}$ normalized voltage is applied on a $140 \Omega$ adapted wave port localized on the antenna input.

Electric field distribution in far field of the system is simulated with CST Microwave Studio software. The electric fields of both vertical and horizontal directions are about $1 \mathrm{~V} / \mathrm{m}$ at $1 \mathrm{~m}$ when $1 \mathrm{~V}$ power is applied to the system, the simulated electric fields distribution is shown in Figure 10. As observed the radiated electric field pulse is a damped sinusoid. The polarization is not perfectly circular, we suppose due to the low number of turns. In these conditions, the antenna factor is about 0.95 .

The simulated beam pattern is provided in Figure 11. The center frequency spectrum of the radiated wave is 


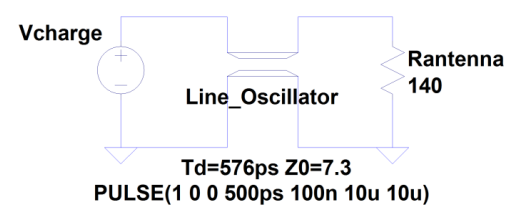

Figure 6. LTspice damped oscillator schematic.

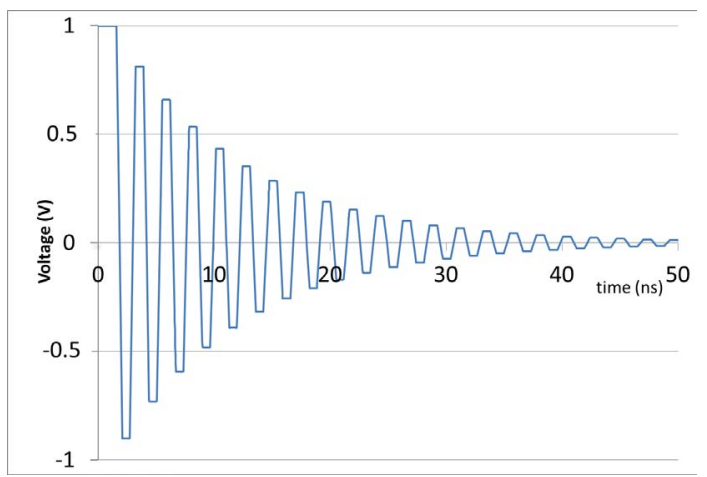

Figure 7. LTspice damped oscillator normalized voltage.

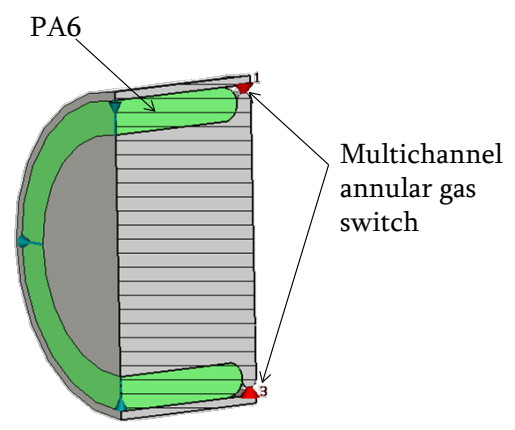

Figure 8. CST damped oscillator model.

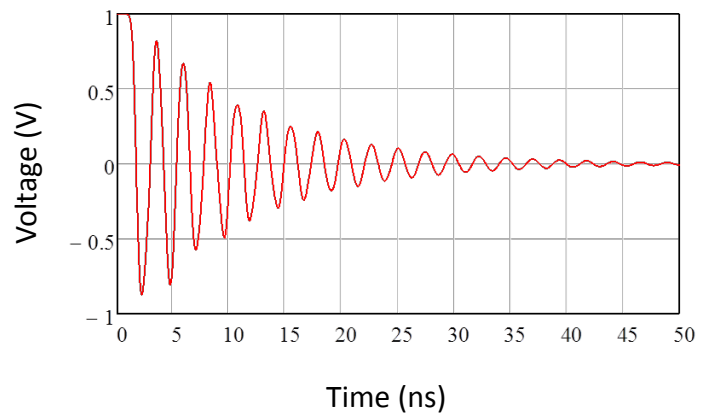

Figure 9. CST damped oscillator normalized voltage.

Table 3. Coaxial damped oscillator design.
Diameter $D_{\text {out }}$ (outer diameter)
Diameter $D_{\text {inn }}$ (inner diameter)
Length $L$
Capacitance $C_{\text {line }}$
Impedance $Z_{\text {line }}$
Propagation time $T_{\text {line }}$
$18.0 \mathrm{~cm}$
$14.3 \mathrm{~cm}$
$9.11 \mathrm{~cm}$
$79.2 \mathrm{pF}$
$7.3 \Omega$
576 ps 


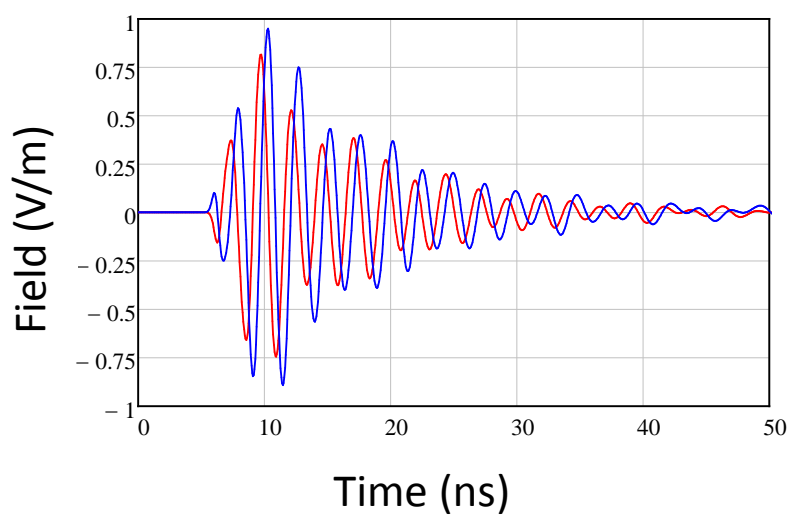

Figure 10. CST electric far fields in $\mathrm{H}$ and $\mathrm{V}$ directions measured at $10 \mathrm{~m}$ and then normalized. Red curve: electric far field in $\mathrm{H}$ direction; Blue curve: electric far field in the $\mathrm{V}$ direction.

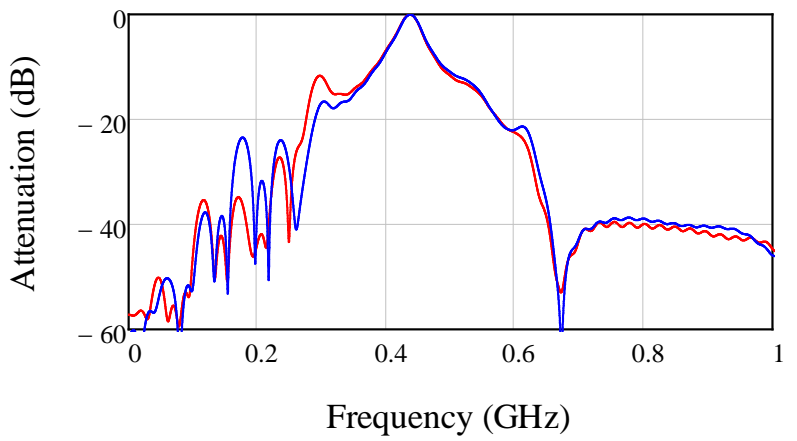

Figure 11. Corresponding spectrums of electric far fields in $\mathrm{H}$ and $\mathrm{V}$ directions measured. Red curve: electric far field in $\mathrm{H}$ direction; Blue curve: electric far field in the $\mathrm{V}$ direction.

close to $434 \mathrm{MHz}$ which is coincide with the center frequency of the transmission line oscillator as seen in Figure 9. The spectral density distribution is between $277 \mathrm{MHz}$ to $575 \mathrm{MHz}$ (at $-20 \mathrm{~dB}$ of the maximum). It was between $65 \mathrm{MHz}$ to $350 \mathrm{MHz}$ with a central frequency spectrum of $212 \mathrm{MHz}$ for the MOUNA source [11]. The proposed changes lead to a slight increase of the bandwidth of the frequency spectrum, and this spectrum is pushed towards high frequencies. This is particularly important in terms of electric susceptibility for HPM systems.

One of the next steps in antenna study is the critical transition between the oscillator and the antenna. This transition (light grey in Figure 12) is the seat of the higher voltage and higher electric field. This point will require a specific study and a specific insulation. To improve radiation performances, Kraus [12] provides a very good example of a cupped helical antenna and this method could be applied in our case.

\section{Conclusions}

A wideband electromagnetic source, named MOUNA and based on a novel high-voltage resonant pulsed transformer, was successfully designed and manufactured. The sub-assembly formed by the novel resonant highvoltage pulsed transformer has been operated up to $600 \mathrm{kV}$ with a rise time of $265 \mathrm{~ns}$. However, to radiate very high electric fields, the low gain of the dipole antenna is detrimental. The use of a directional antenna may improve performances really significantly focusing the radiation in a preferred direction. In order to improve the overall electrical performances, two approaches were explored in the paper: the replacement of the dipole antenna by a helical antenna and its feeding signal influence. The theoretical simulation comparison between various feeding signals applied to a helical antenna points out a strong dependence of the shape of the radiated field with the shape of the feeding signal. The highest antenna factor is obtained with a damped sinusoid. Moreover the impulse feeding directly integrated in the antenna design improves both the overall dimensions and electromagnetic characteristics [23]. 


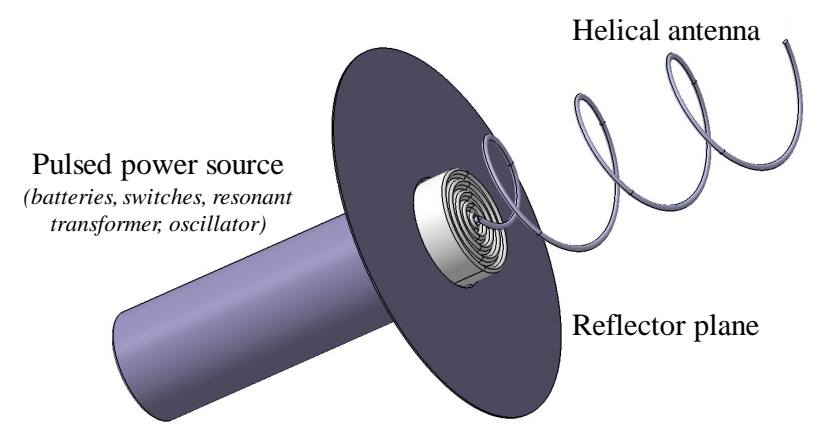

Figure 12. Schematic view of SPIRALE power source.

The concept of a switched oscillator as a highly capacitive quarter-wave transmission line terminated by an antenna load of high input impedance on one end and a self-breaking switch on the other end is proposed. After the switch closes, the stored energy is discharged into the high impedance load with a waveform that resembles a damped sinusoid. The predicted results concerning the design of the helical antenna, of a switch oscillator directly implemented at the output of the transformer have been presented. Simulation of the whole system is a useful way to predict electrical characteristics in the initial stage of manufacturing processes. This CST-based field simulation confirms the best expected performances. The antenna factor is 0.95 which allows a complete source with a very high figure of merit. In the present study the impedance of the closed switch has been assumed negligible. It is difficult to predict the rise time of the annular switch. However, it will have some inductance, lowering the resonant frequency, and some resistance, increasing the damping of the resonance. These effects need to be minimized. More accurate models can be developed to more precisely compute the performance. Probably the unknown losses in the circuit and particularly the performances of the switch will alter the calculated figure-of-merit.

The pulsed source with its new damped oscillator contains a volume of only 2500 cc. To achieve such compactness, all these parts are integrated within the antenna and immersed in oil. To keep such compactness, the helical antenna could be deployed in operational situation. The source performances could be increased by working on the reflector geometry or on the number of turns. Particularly a cupped ground plane could be implemented to improve the electrical performances.

\section{Acknowledgements}

These works are driven and financially supported by the Atomic Energy Commission (CEA), contract No. 4600233705.

\section{References}

[1] Giri, D.V. (2004) High Power Electromagnetic Radiators: Nonlethal Weapons and Other Applications. Harvard University Press, Cambridge.

[2] Morton, D., Banister, J., Levine, J., Naff, T., Smith, I., Sze, H., Warren, T., Giri, D., Mora, C., Pavlinko, J., et al. (2010) A $2 \mathrm{MV},<300$ ps Risetime, $100 \mathrm{~Hz}$ Pulser for Generation of Microwaves. Proceedings of the 2010 IEEE International Power Modulator and High Voltage Conference (IPMHVC), Atlanta, 23-27 May 2010, 361-364. http://dx.doi.org/10.1109/ipmhvc.2010.5958368

[3] Koshelev, V.I., Plisko, V.V. and Sukhushin, K.N. (2010) Array Antenna for Directed Radiation of High-Power Ultra-wideband Pulses. In: Sabath, F., Giri, D.V., Rachidi, F. and Kaelin, A., Eds., Ultra-Wideband, Short Pulse Electromagnetics 9, Springer, New York, 259-267.

[4] Cadilhon, B., Pecastaing, L., Vauchamp, S., Andrieu, J., Bertrand, V. and Lalande, M. (2009) Improvement of an Ultra-Wideband Antenna for High-Power Transient Applications. IET Microwaves Antennas and Propagation, 3, 11021109.

[5] Cadilhon, B., Cassany, B., Diot, J., Modin, P., Merle, E., Pecastaing, L., Rivaletto, M., Silvestre de Ferron, A. and Bertrand, V. (2011) Self-Contained, Hand-Portable, and Repetitive Ultrawideband Radiation Source. IEEE Transactions on Plasma Science, 39, 1549-1559. http://dx.doi.org/10.1109/TPS.2011.2135867

[6] Cadilhon, B., Pecastaing, L., Reess, T., Silvestre de Ferron, A., Pignolet, P., Vauchamp, S., Andrieu, J. and Lalande, M. 
(2010) High Pulsed Power Sources for Broadband Radiation. IEEE Transactions on Plasma Science, 38, 2593-2603. http://dx.doi.org/10.1109/TPS.2010.2042732

[7] Delmote, P., Dubois, C., Andrieu, J., Beillard, B., Lalande, M., Bertrand, V., Jecko, B., Pecastaing, L., Gibert, A., Paillol, J., Domens, P., Guillerey, R., Monnier, F. and Legoff, M. (2003) UWB SAR System PULSAR: New Generator and Antenna Developments. Proceedings of SPIE 5077, Passive Millimeter-Wave Imaging Technology VI and Radar Sensor Technology VII, 25 August 2003, 223-234.

[8] Radasky, W.A., Baum, C.E. and Wik, M.W. (2004) Introduction to the Special Issue on High-Power Electromagnetics (HPEM) and Intentional Electromagnetic Interference (IEMI). IEEE Transactions on Electromagnetic Compatibility, 46, 314-321. http://dx.doi.org/10.1109/TEMC.2004.831899

[9] Stark, R. (2011) Diehl HPEM—Source Development. The High Power Microwave Defense and Security Workshop, Saint-Louis.

[10] Hong, K.D. and Braidwood, S.W. (2002) Resonant Antenna-Source System for Generation of high-Power Wideband Pulses. IEEE Transactions on Plasma Science, 30, 1705-1711. http://dx.doi.org/10.1109/TPS.2002.806637

[11] Pecquois, R., Pecastaing, L., Rivaletto, M., Silvestre De Ferron, A. and Vézinet, R. (2012) MOUNA: An Autonomous, Compact, High-Power, and Wideband Electromagnetic Source Based on a Novel Resonant Pulsed Transformer. IEEE Transactions on Plasma Science, 40, 1407-1415. http://dx.doi.org/10.1109/TPS.2012.2189138

[12] Kraus, J. (2002) Antennas for All Applications. 3rd Edition, McGraw-Hill, New York.

[13] Carey, W., Wiebe, A., Schwindt, D., Altgilbers, L., Giesselmann, M., McHale, B. and Heinemann, K. (2005) Autonomous RF Radiation Package for Various Applications. Proceedings of the 2005 IEEE Pulsed Power Conference, Monterey, 13-17 June 2005, 218-221. http://dx.doi.org/10.1109/ppc.2005.300564

[14] Mayes, J.R., Mayes, M.G., Nunnally, W.C. and Hatfield, C.W. (2009) Helical Antennas for High Powered RF. Proceedings of the 2009 Pulsed Power Conference, Washington DC, 28 June-2 July 2009, 484-488. http://dx.doi.org/10.1109/ppc.2009.5386324

[15] Lee, J.M., Kwon, H.O., Hwang, S.M. and Ahn, J.W. (2011) Design of a Damped Sinusoidal Oscillator System. Proceedings of the 2011 IEEE Pulsed Power Conference, Chicago, 19-23 Jun 2011, 414-419. http://dx.doi.org/10.1109/ppc.2011.6191456

[16] Mosfegh, J., Shahabadi, M. and Rashed-Mohassel, J. (2011) Conditions of Maximum Efficiency for Wireless Power Transfer between Two Helical Wires. IET Microwaves Antennas and Propagation, 5, 545-550.

[17] Sabath, F., Giri, D.V., Rachidi-Haeri, F. and Kaelin, A., Eds. (2010) Ultra-Wideband, Short Pulse Electromagnetics 9. Springer, New York, 315-324.

[18] Giri, D.V., Tesche, F.M., Abdalla, M.D., Skipper, M.C. and Nyffeler, M. (2010) Switched Oscillators and Their Integration Into Helical Antennas. IEEE Transactions on Plasma Science, 38, 1411-1426. http://dx.doi.org/10.1109/TPS.2010.2047657

[19] Armanious, M. and Tyo, J.S. (2010) Field Management for a Self-Breakdown Switched Oscillator. Proceedings of the IEEE International Symposium on Antennas and Propagation Society (APSURSI), Toronto, 11-17 July 2010, 1-4. http://dx.doi.org/10.1109/aps.2010.5562335

[20] Baum, C.E. (2000) Switched Oscillators. Circuit and Electromagnetic System Design Note 45.

[21] Pecastaing, L., Reess, T., Paillol, J., Gibert, A. and Domens, P. (2001) Electrical Breakdown Studies of Pressurised Nitrogen in Non-Uniform Fields. European Physical Journal Applied Physics, 16, 59-69. http://dx.doi.org/10.1051/epjap:2001194

[22] Pecquois, R., Pecastaing, L., De Ferron, A., Rivaletto, M., Pignolet, P., Novac, B.M., Smith, I.R. and Adler, R.J. (2012) Simple and Compact Capacitive Voltage Probe for Measuring Voltage Impulses up to $0.5 \mathrm{MV}$. Review of Scientific Instruments, 83, Article ID: 035001. http://dx.doi.org/10.1063/1.3690906

[23] Bertrand, V., Negrier, R., Lalande, M., Andrieu, J., Couderc, V., Shalaby, B., Pecastaing, L. and De Ferron, A. (2014) Equivalent Model of Photoswitch: Application to the UWB Antenna Design Integrating Impulse Feeding. Progress in Electromagnetic Research C, 46, 145-151. http://dx.doi.org/10.2528/PIERC13120301 